Revue d'histoire de l'enfance « irrégulière »

Le Temps de l'histoire

Hors-série | 2001

Histoire et justice, panorama de la recherche

\title{
Le vol nécessaire au XIXème siècle. Entre réalité sociale et lacune juridique, une histoire en construction.
}

Virginie Berger

\section{(2) OpenEdition \\ Journals}

Édition électronique

URL : http://journals.openedition.org/rhei/462

DOI : $10.4000 /$ rhei. 462

ISBN : 978-2-7535-1641-0

ISSN : $1777-540 \mathrm{X}$

Éditeur

Presses universitaires de Rennes

Édition imprimée

Date de publication : 15 novembre 2001

Pagination : 241-251

ISSN : $1287-2431$

Référence électronique

Virginie Berger, «Le vol nécessaire au XIXème siècle. Entre réalité sociale et lacune juridique, une histoire en construction. », Revue d'histoire de l'enfance "irrégulière » [En ligne], Hors-série | 2001, mis en ligne le 31 mai 2007, consulté le 20 avril 2019. URL : http://journals.openedition.org/rhei/462 ; DOI : $10.4000 /$ rhei.462 


\section{Le vol nécessaire au XIXème siècle. Entre réalité sociale et lacune juridique, une histoire en construction.}

Il est frappant de constater qu'un phénomène, aussi présent dans notre société et aussi controversé que celui du vol, ait été longtemps sous-estimé par les historiens. Le silence entourant cette catégorie d'infraction est d'autant plus surprenant qu'il s'agit du contentieux le plus fréquemment traité devant la justice et considéré pendant longtemps comme plus dangereux que les atteintes aux personnes. ${ }^{(2)}$ Au premier abord, il est vrai, le vol s'avère difficile à appréhender, tant par le volume d'affaires judiciaires qu'il représente, que par la multitude des cas de figure qu'il revêt. Cependant, ne pas étudier ce phénomène revient à occulter une partie de l'histoire sociale et judiciaire de la France, laissant dans l'ombre une part importante de la criminalité jugée par les tribunaux.

Parmi les différents cas de figure du vol, la soustraction commise en état de nécessité semble la moins connue, malgré l'existence d'une bibliographie juridique sur le sujet. ${ }^{(3)}$ Cette situation s'explique probablement par l'introduction tardive dans le Code pénal de la notion de nécessité. Ce n'est, en effet, qu'en 1994 avec la promulgation du nouveau Code ${ }^{(4)}$ que l'idée d'acte nécessaire est véritablement entérinée dans notre droit, venant ainsi combler une lacune concernant une notion pourtant connue depuis l'antiquité et acceptée dans l'ancien droit. Aujourd'hui encore, en dépit de cette reconnaissance, le vol nécessaire semble toujours poser quelques problèmes aux magistrats. L'actualité en témoigne par l'agitation médiatique et judiciaire suscitée, il y a quelques années, au sujet de la relaxe prononcée par le tribunal correctionnel de Poitiers en faveur d'une femme, aux revenus modestes, qui avait reconnu le vol de plusieurs morceaux de viande pour nourrir ses deux enfants, et qui avait invoqué, pour sa défense, l'état de nécessité. ${ }^{(5)}$

\section{Virginie \\ Berger $^{(1)}$}

(1) Doctorante en histoire contemporaine à l'université de Poitiers.

(2) Michelle PERROT, "Délinquance et système pénitentiaire en France au XIXème siècle”, Annales ESC, Paris, 1975, p. 73.

(3) Voir en particulier : Paul MORIAUD, De la justification du délit par l'état de nécessité, thèse de doctorat de droit, Genève, Paris, 1889 , 319 p. ; J.A. ROUX, "Rapport sur l'état de nécessité et le délit nécessaire" et "Discussion du rapport Roux”, Revue pénitentiaire et de droit pénal, tome XXIV, 1900, pp. 1411-1450; tome XXV, 1901, pp. 48-89; LEREBOURS-

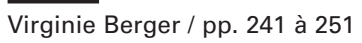


PIGEONNIÈRE Paul,

"Rapport de la première section sur l'état de nécessité”, Revue pénitentiaire et de droit pénal, tome XXV, 1901, pp. 329-338 ; DOUCET Robert, "La répression des délits causés par la misère", Revue politique et parlementaire, tome XXV, juillet 1900, pp. 88-94 ; FORIERS Paul, De l'état de nécessité en droit pénal, Paris, 1951 ; CHEVALIER Jean-Yves, L'état de nécessité, Mélanges en l'honneur du doyen Pierre Bouzat, Paris, A. Ledone, 1980, pp. 117-134.

(4) Notion introduite par l'article 122-7 du nouveau Code pénal : " n'est pas pénalement responsable la personne qui face à un danger actuel ou imminent, qui menace ellemême, autrui ou un bien, accomplit un acte nécessaire à la sauvegarde de la personne ou du bien, sauf s'ily a disproportion entre les moyens employés et la gravité de la menace. "
Vouloir étudier le vol nécessaire, en France pour la période du XIXème siècle, peut sembler relever d'une véritable gageure puisque la nécessité n'est nullement mentionnée dans la codification pénale de ce siècle. Cependant, une telle étude s'avère envisageable au regard de plusieurs éléments. En effet, comment considérer l'association des concepts de vol et de justification par la nécessité, qui du point de vue juridique sont littéralement antagoniques puisque mettant en opposition deux notions fondamentales : celle de la propriété et celle de l'acte nécessaire ? La codification des lois établie au début du siècle, ne laissant encore qu'une faible place à la jurisprudence, la nécessité reste un problème entier pour les juristes du XIXème siècle. Ainsi, ce thème de la nécessité dans la législation n'a cessé tout au long du siècle d'alimenter de nombreux débats entre les divers théoriciens et praticiens du droit français. Cette agitation de la "sociétéjudiciaire » est révélatrice de l'existence d'un profond malaise présent, non seulement dans la sphère juridique, mais se ressentant également dans l'ensemble de la société. Ceci est à mettre en relation avec les représentations très fortes qui sont associées au vol à cette époque. En effet, on assiste à une véritable stigmatisation autour du vol qui est alors perçu par la société comme un "fléau ", menaçant la base même de son organisation sociale : le droit à la propriété. Ce siècle offre donc un terrain d'étude propice au traitement par l'historien de certains aspects propres au vol appliqué à l'état de nécessité ; aspects qui n'ont été jusqu'à présent étudiés que du point de vue juridique. Un autre point semble très intéressant à soulever, c'est celui de la connaissance des voleurs. Qui sont-ils ? Pourquoi enfreignent-ils la loi ? Comment étudier ces voleurs?

L'historien a donc un rôle primordial à jouer dans la mise en avant de cet aspect de l'activité judiciaire. En effet, le vol nécessaire, aussi minime soit-il dans la pratique judiciaire, n'en reste pas moins un élémentclé dans la compréhension d'une certaine réalité sociale propre au XIXème siècle.

Au delà de la simple infraction commise par nécessité, le vol nécessaire bouscule un des fondements de cette société, aussi bien juridique- 
ment que philosophiquement. Les passions, les discussions accompagnant le vol nécessaire au cours de ce siècle se posent comme autant de révélateurs d'un changement symptomatique des sensibilités.

Pour mieux comprendre ce qu'est le vol nécessaire, il est préférable de définir dans un premier temps les deux notions le constituant, c'est-àdire, le vol et la nécessité.

Le vol se définit comme la soustraction frauduleuse d'une chose appartenant à autrui, ${ }^{(6)}$ constituant une violation de la loi pénale et impliquant inévitablement, dans l'inconscient collectif, un châtiment ; toute infraction à la loi se devant d'être punie pour que soit garanti l'ordre public. ${ }^{(7)}$ Cependant, il existe des faits justificatifs qui, lorsqu'ils sont présents, suppriment la responsabilité pénale de l'auteur de l'infraction. ${ }^{(8)}$ Non pas que ces faits supposent l'irresponsabilité de l'agent au moment de l'infraction, mais ils impliquent une justification des faits par la loi elle-même.

Aujourd'hui, en droit pénal, l'acte nécessaire fait partie intégrante des éléments qui composent la théorie de la responsabilité pénale. Il est considéré comme un fait justificatif au même titre que la légitime défense. ${ }^{(9)}$ Il est défini comme la situation dans laquelle un individu, pour sauver un bien ou un intérêt, commet une infraction portant préjudice à une personne innocente. ${ }^{(10)}$ L'intérêt de la société est alors placé au-dessus de l'intérêt privé, si le préjudice causé est moindre que celui évité. Dans une pareille situation de nécessité, l'agent en état de nécessité est placé devant une collision de devoirs, un conflit opposant deux intérêts antinomiques, l'obligeant à faire le choix de l'un au détriment de l'autre. Cette liberté de décision implique une prise de conscience de la part de l'agent de l'illégalité du geste qu'il va commettre.

Cette théorie repose sur des fondements historiques très anciens. Déjà en droit romain, l'état de nécessité était retenu comme une cause d'irresponsabilité. L'impunité de l'infraction était fondée sur le mobile non criminel de l'individu qui vole, c'est-à-dire le défaut d'intention délictueuse, appelée dol par les romains. Le droit germanique reconnaissait, lui aussi, la nécessité ; elle était alors perçue à la fois comme un motif et
(5) Audience du

5 février 1997 du tribunal correctionnel de Poitiers. L'affaire a été suivie par le quotidien Le Monde et a fait l'objet de plusieurs articles : Le Monde des 28 février 1997, 1er mars 1997, 3 mars 1997, 4 mars 1997, 31 mars et du 12 avril 1997.

(6) Article $379 \mathrm{du}$ Code pénal :

"Quiconque a soustrait une chose qui ne lui appartient pas est coupable de vol."

(7) Robert DOUCET, op. cit., p. 92 : «Le premier et unique but de toute notre législation est de protéger les citoyens et de punir ceux qui troublent l'ordre public et social. "

(8) Ils ne suppriment cependant pas la responsabilité civile.

(9) Pierre BOUZAT, Jean PINATEL, Traité de droit pénal et de criminologie, tome I, 2ème édition, Paris, Libraire Dalloz, 1970, p. 367. 
(10) Cette dernière mention permet notamment de différencier la légitime défense de l'état de nécessité

(11) Idée défendue notamment par GROTIUS dans Le Droit de la guerre et de la paix, tome 1, livre II, PradierFodéré, p. 401 et ss.

(12) Thèse soutenue par PUFENDORF dans Le Droit de la nature et des gens, traduction du latin par J. Barbeyrac, Bâle, 1732.

(13) André LANGUI, Arlette LEBIGRE,

Histoire du droit pénal, tome I, Le droit pénal, Cujas, Paris, 1979, pp. 107-110.

(14) Ces documents sont conservés dans la série $2 \mathrm{U}$ des Archives départementales; ils peuvent être complétés par la série BB des Archives nationales.

(15) Virginie BERGER, Un vol de nécessité: le vol d'aliments dans la Vienne dans la première une cause de la justification de l'auteur de l'infraction. Il serait d'ailleurs à l'origine du célèbre adage : "nécessité n’a pas de loi ». En ce qui concerne le droit canonique, la nécessité conservait, dans la lignée du droit germanique, son caractère justificatif, l'impunité de l'infraction était conçue sur une idée de solidarité et de miséricorde. Au Moyen Âge, le pauvre bénéficiait encore de la tradition évangélique qui voyait en lui l'envoyé de Dieu. D'autres fondements, d'ordre philosophique ceux-ci, prônés notamment par les philosophes du droit naturel, reposaient sur une idée de retour à l'état de nature ${ }^{(11)}$ et à un instinct de conservation irrésistible. $^{(12)}$ Il faut souligner que l'impunité du vol nécessaire était encore reconnue par les criminalistes des XVIIème et XVIIIème siècles, comme Muyart de Vouglans, Rousseau de la Combe et Jousse.(13)

Dans l'optique de son application au vol, l'état de nécessité s'impose, par exemple, lorsqu'une soustraction a été accomplie pour repousser un danger imminent menaçant la sécurité de son auteur ou de tiers. Le cas le plus emblématique de cette situation est le vol d'aliments commis sous l'empire de la misère. Les archives judiciaires des cours d'assises ${ }^{(14)}$ renferment plusieurs cas de vols commis dans ces circonstances. Qu'ils soient simples journaliers ou petits artisans, ces voleurs sont des gens ordinaires, souvent un voisin, appartenant à la communauté villageoise. ${ }^{(15)}$ Ils sont très éloignés de l'image des brigands transmise par la littérature judiciaire qui se développe à l'époque. Vivant à la limite de la pauvreté, ils sont à la merci des mauvaises conditions climatiques pour se nourrir et de la conjoncture économique pour se procurer du travail. Cependant, ce ne sont pas ce que l'on peut appeler des voleurs de profession, de "francsvoleurs $^{(16)}$ » vivant de leurs forfaits. Le passage à l'acte de ces voleurs trahit une rupture, un seuil de tolérance qui n'est plus supportable. Ils savent qu'ils risquent gros pour un morceau de pain ramené à leur famille. Ainsi François Plourde, journalier à Thuré, petite commune rurale de la Vienne, reconnaît devant les assises du département de la Vienne, avoir volé quatre pains de froment dans une boulangerie pour nourrir sa femme et ses enfants qui n'avaient rien à manger depuis plusieurs jours. ${ }^{(17)}$ Reconnu coupable par les jurés, il est condamné le 17 août 1831 à cinq 
ans de travaux forcés par la cour d'assises. ${ }^{(18)}$ La " misère ", le " grand besoin ", "l'extrême dénuement" sont des termes récurrents qui se retrouvent dans les affaires de vols qui nous intéresse. ${ }^{(19)}$

Une étude poussée, de type anthropologique, des voleurs semble donc une voie intéressante pour mieux comprendre les tenants et les aboutissants du vol nécessaire. Les archives judiciaires restent alors une source d'information irremplaçable pour un tel traitement quoique limitée. ${ }^{(20)}$

Les affaires fournissant de plus amples détails concernant les voleurs, leur vie et leurs comportements sont rares mais pas inexistantes. Voici des extraits de l'interrogatoire de Jean Bonnet, un journalier de trenteneuf ans, né à Luchapt, une petite commune rurale du département de la Vienne, qui relatent les événements qui l'ont amené à commettre un vol $^{(21)}$ en janvier 1846. Ce document est particulièrement précieux pour l'historien car il permet de retracer l'emploi du temps du voleur, les démarches accomplies par celui-ci pour trouver de quoi se nourrir : «Le lundi 5 janvier, j'étais allé chez un nommé Faura au lieu de Chassou, commune de Luchapt, réclamer une somme de cinq francs qu'il me devait depuis trois ou quatre ans pour une faux que je lui ai vendu, ne l'ayant point trouvé je me rendis chez Jean Pailler, meunier, réclamer trois francs qu'il me devait pour faiçon d'un cent de fagote [sic]. Il n'avait ni argent, ni blé à me donner. "

L'année 1846 est particulièrement dure puisqu'il s'agit d'une année de crise céréalière, due à de mauvaises conditions climatiques, ayant entraîné un phénomène de disette. ${ }^{(22)}$ Jean Bonnet n'est pas tombé dans la misère de son fait, il compte fortement sur la solidarité villageoise pour sortir de cette mauvaise passe. Il exprime son état d'esprit lorsqu'il se trouve en face de sa famille, sans un sou, sans un morceau de pain à leur donner. La famille joue un rôle très important dans les affaires de vol nécessaire, elle détermine bien souvent le passage à l'acte du voleur : "Je sortis ensuite, il pouvait être sept ou buit heures du soir, je ne saurais pas précisément vous indiquer l'beure, mais il était nuit depuis quelques heures, je rentrai chez moi, ma femme et mes enfants se mirent à crier sur moi en demandant du pain disant qu'ils avaient faim. Le cour me saignait de douleur de les voir ainsi crier la faim sans pouvoir les soulager. " moitié du XIXème siècle, mémoire de maîtrise (sous la direction de Frédéric Chauvaud), université de Poitiers, 1999, $110 \mathrm{f}^{\circ}$.

(16) Archives du département de la Vienne, 2U 1508. Terme utilisé par un voleur interrogé sur son crime, un vol de pain, par le juge d'instruction près le tribunal correctionnel de Civray, le 25 juillet 1831.

(17) Archives départementales de la Vienne, 2U 1507, année 1831, dossier sans numéro. Extrait de l'interrogatoire de François Plourde du 4 juin 1831 : «Cen'est que la misère et parce qu'il n'y avait point de pain chez moi pour ma femme et mes petits enfants qui pleuraient qui m'a porté à commettre cette action."

(18) Avant avril 1832, le jury n'a pas la possibilité d'accorder les circonstances atténuantes ; seule la cour possède cette faculté depuis la loi 
de 1824 .

(19) Archives départementales de la Vienne, liasse 2U 1511, année 1832, dossier $n^{\circ} 959$. Liasse 2 U 539, année 1818, dossier $n^{\circ} 271$. Liasse 2U 1526, année 1836, dossier n 1204 : interrogatoire $\mathrm{du}$ 17 octobre 1836. Liasse 2U 1508, année 1831, dossier sans numéro, affaire Louis Dury. Archives départementales des Deux-Sèvres, liasse 2U 194, année 1846, dossier n 13 .

Liasse 2U 166, année 1838 , dossier $n^{\circ} 860$.

(20) Virginie BER-

GER, Le vol ou la misère, la nécessité de survivre dans le Poitou au XIXème siècle, mémoire de DEA (sous la direction de Frédéric Chauvaud), université de Poitiers, 2000, $134 f^{\circ}$.

(21) Archives départementales de la Vienne, 2U 1572, dossier 1856, interrogatoire du 17 janvier 1846.

(22) René MEMAIN,
Mais peut être plus que sa famille, c'est sûrement le refus de son boulanger de lui faire crédit d'un morceau de pain et le risque de se voir présenter un nouveau refus de la part du meunier qui lui fait franchir le pas. Il n'est pas rare en effet de trouver ce genre de déclaration dans d'autres affaires ${ }^{(23)}$ : "Je me saisis d'un sac pour aller chez le boulanger nommé Barland demander du pain à emprunter. Je ne trouvai que la femme et la domestique qui me dirent que je n'aurais point de pain sans argent. Je sortis dans l'intention de me rendre au moulin de la Lange chez le nommé Jean Pailler, parent de l'autre Pailler, pour demander du blé ou de la farine. »

Face au refus de l'entraide villageoise, l'envie de se servir soi-même s'impose au voleur et semble une solution plus facile mais surtout apportant un résultat sûr et immédiat : des denrées pour nourrir ses enfants. L'interrogatoire laisse à penser que Bonnet avait conscience de commettre une mauvaise action, qu'il savait qu'il enfreignait la loi : "Passant près d'une petite maison séparée de toute habitation et inhabitée. Il me vint une mauvaise pensée dans l'esprit. Je perdis la tête. J'eus le malheur de monter à l'aide d'un morceau de bois le long du mur à la croisée de la maison de M. Lacouture, cette croisée peut être élevée du sol d'environ trois mètres. Je saisis avec la main le barreau en bois qui se trouvait à cette croisée. Il était pourri et il céda facilement. Je pénétrai par cette croisée dans le grenier où je pris une petite quantité de mauvaises feves et ensuite je levai le sac par le milieu et mis un peu de colza dans le surplus du sac vide. J'emportai ensuite le tout à la maison, je fis cuire un peu des feves pour apaiser la faim de mes enfants, je cachai le surplus dans le jardin dans des fagots ainsi que le colza. »

Il ne faut pas oublier que le vol de nourriture est provisoire, il ne suffit pas pour sortir d'une période de crise. Le comportement de Bonnet après sa soustraction, démarchant à nouveau pour trouver de la nourriture, montre que le vol qu'il a commis n'était qu'une échappatoire exceptionnelle. Conscient de son geste, il s'en remet à la justice non sans avoir demandé l'avis du maire de sa commune, intermédiaire primordial dans ces affaires : «Le lendemain je me rendis chez Pailler mon meunier qui me vendit à crédit un double décalitre de froment et un double décalitre de seigle, tout est mangé, je ne l'ai point payé et mes enfants et ma fermme se trouvent dans la plus 
grande des misères. Mercredi dernier quatorze janvier, la gendarmerie se présenta chez moi, j'étais absent, je ne rentrai que le jeudi soir et j'appris que la gendarmerie était venue me chercher. Vendredi matin 16, je fus trouver le maire qui me conseilla de me rendre à la gendarmerie de l'Isle Jourdain. Ce que je fis. "

Jugé devant la cour d'assises du département de la Vienne, Jean Bonnet est acquitté par les jurés qui n'ont pas voulu condamner cet homme. Peut-être ne doit-on voir dans cet acquittement qu'un simple acte de mansuétude, de miséricorde de la part des jurés, mais n'est-il pas envisageable d'y saisir une reconnaissance tacite de l'état de nécessité ? Car il faut reconnaitre que cette affaire regroupe tous les traits de définition accordés aujourd'hui à l'état de nécessité.

Un tel acquittement ne laisse toutefois pas indifférent le président de cette session des assises de la Vienne qui exprime, dans son rapport de session à la Chancellerie, son sentiment sur l'affaire : "Il avouait le fait et les circonstances. Cependant le jury l'a déclaré non coupable. Il avait volé un double décalitre de colza et une quantité égale de feves. Son excellente réputation jusque là et son extrême misère, attestée par le maire de la commune, expliquent cet acquittement, mais ne le justifient pas. ${ }^{(24)}$ " "Ne le justifie pas" : à eux seuls, ces quelques mots résument la pensée dominante des représentants de la justice du XIXème siècle. En effet, selon le Code pénal, rien ne peut justifier la commission d'une soustraction.

Le travail de l'historien permet d'analyser et de comprendre le rapport qui se met en place et évolue au cours du XIXème siècle entre les juristes et la théorie du vol nécessaire. La dimension anthropologique est à même de compléter les travaux des juristes et des historiens du droit dans l'analyse de cette relation.

Après la Révolution, l'idée de délit nécessaire tombe progressivement dans l'oubli. Le Code pénal de 1810 ne fait, en effet, aucune référence à l'état de nécessité en dehors des cas précis prescrits par les articles 327 et 328. ${ }^{(25)}$ Le seul article faisant disparaître totalement la responsabilité pénale est l'article 64 du Code qui stipule : "Il n'y a ni crime, ni délit,
La crise de 1845-1847

dans la Vienne, SAO,

4ème série, tome $\mathrm{V}$,

1er trimestre 1960,

pp. 475-523 et 3ème tri-

mestre 1960, pp. 547-

615.

(23) Archives départementale de la Vienne 2U 1505, dossier Dury, vol en juillet 1831 : «Je répète que la misère seule m'a fait commettre un vol et que j'ai principalement été excité par le refus de mon boulanger de me donner $d u$ pain."

(24) Archives nationales BB 20/136 :

Rapport du président des assises du département de la Vienne adressé à la Chancellerie pour le premier trimestre 1846.

(25) Articles du Code pénal qui exonèrent de toute répression des délits exécutés sous ordre de l'autorité légitime. 
(26) Adolphe CHAU-

VEAU, Faustin HELIE, Théorie du Code pénal, Tome 1, Paris, Cosse \& Co., 1872 , p. 570 .

(27) Paul MORIAUD, op. cit. lorsque le prévenu était en état de démence au moment du fait, ou lorsqu'il a été contraint par une force à laquelle il n'a pu résister. " Il est fait ici référence à la théorie de la contrainte utilisée dans son acception la plus large et déjà présente chez certains criminalistes du XVIIIème siècle. Cette théorie se base sur la perte du libre arbitre de l'auteur de l'infraction lorsque celui-ci est exposé à un danger imminent. Or, l'existence d'une infraction en droit est fondée sur la responsabilité et la liberté de son auteur ; sans cela l'infraction n'existe pas. La nécessité a longtemps été assimilée à l'idée de contrainte. Cependant cette théorie a été, par la suite, largement critiquée dans son principe même, puisque comme nous l'avons évoqué ci-dessus, l'état de nécessité ne supprime pas le libre arbitre de l'auteur de l'infraction. Ces deux notions ne sont donc pas équivalentes et la nécessité incarne bien une véritable lacune juridique.

En théorie, la nécessité n'apparaît pas dans le Code. Mais qu'en est-il dans la pratique ? Quelle interprétation les juristes ont-il fait des textes? Si l'on se réfere aux théoriciens et commentateurs du Code pénal de la seconde moitié du XIXème siècle, il semble que le silence des textes ait été interprété par les juristes comme une négation de l'état de nécessité. Ainsi, Adolphe Chauveau et Faustin Hélie rejettent purement l'état de nécessité comme justificatif possible de l'infraction. Ils affirment que "la faim et la misère sont bien des circonstances atténuantes des délits, mais qu'il serait immoral et dangereux de proclamer dans ce cas la justification complète de l'agent, car ce serait reconnaître que les règles de la justice morale ont leurs limites et leurs exceptions ${ }^{(26)}$ ". Pour appuyer leur position, ils ajoutent que l'article $64 \mathrm{du}$ Code ne reconnaît ni dans la misère, ni dans la faim, la force irrésistible qui peut justifier le crime. Ils se retranchent derrière le Code pénal représentant tout le Droit et considéré alors comme parfait.

Cet état de fait contribue à la faible diffusion de la théorie de la nécessité en France durant la plus grande partie du XIXème siècle. Les véritables réflexions faites sur le concept de l'état de nécessité datent de la fin du XIXème siècle, avec notamment celles de Paul Moriaud développées dans une thèse intitulée $D u$ délit nécessaire et de l'état de nécessité, (27) publiée en 1889. Dans cet ouvrage, l'auteur s'appuie sur la carence du droit fran- 
çais pour développer ses théories sur le principe de la nécessité. Paul Moriaud passe en revue les diverses législations et doctrines ayant existé depuis l'Antiquité ; il étudie aussi celles en vigueur au XIXème siècle, et en propose une critique constructive. L'apport majeur de cet ouvrage est, sans doute, la contestation faite sur le principe de la contrainte morale, prônée durant le siècle, et avancé par la jurisprudence pour les cas de nécessité. Mais Paul Moriaud ne s'arrête pas là, il s’intéresse également à la théorie même de l'état de nécessité, en abordant, notamment, les principes, les restrictions et les conditions d'impunité qui doivent y être liées. Ces idées, connues par quelques praticiens du droit, ${ }^{(28)}$ entraînent un changement de la pensée juridique au sujet de la nécessité.

Peut-être influencées par ces écrits, c'est également à la fin du siècle que des voix s'élèvent, parmi la magistrature, face au silence du Code pénal. Le juge Magnaud, président du tribunal de Château-Thierry, surnommé "le bon juge ${ }^{(29)}$ ", s'est fait remarquer par plusieurs acquittements prononcés en faveur des plus démunis. Il a notamment défrayé la chronique en 1898 en prononçant un jugement d'acquittement à l'égard de Louise Ménard, une voleuse de pain, ayant agi sous l'empire de la faim et dans l'intérêt de la survie de sa famille. ${ }^{(30)}$

Ce jugement est accueilli très favorablement par la presse. Il est relayé au niveau national comme un jugement " bumain ». Cependant, quelques voix s'élèvent et dénoncent la création d'un terrible précédent : le Droit au vol. ${ }^{(31)}$ C'est en effet à partir de cette affaire, qui favorise le dépôt d'une proposition de loi ${ }^{(32)}$ portant sur la modification de l'article $64 \mathrm{du}$ Code et la prise en compte de l'état de nécessité, que se multiplient les réflexions sur le délit nécessaire et l'état de nécessité. Les plus brillants et éminents spécialistes du droit et de la jurisprudence se penchent sur le sujet et expriment leurs opinions, soit par l'intermédiaire de revues savantes ou bien de discours prononcés devant les corps professionnels judiciaires. ${ }^{(33)}$ Ces débats interviennent dans une période où l'on pense qu'une refonte de la législation pénale, cent ans après son élaboration, semble nécessaire pour aboutir à la création d'une nouvelle œuvre prenant en compte les divers changements intervenus dans la société.
(28) M. MAR-

CHAND, avocat général de la cour d'appel de Nancy, y fait notamment référence lors d'un discours de rentrée de la cour portant sur le vol en cas d'extrême misère et l'état de nécessité, prononcé le 16 octobre 1900 à Nancy, impr. de Vagner, Nancy, 1900, p. 11 ; J.-A. ROUX, op. cit.

(29) Le président

Magnaud doit son surnom à Georges Clemenceau qui lui consacra un article concernant le jugement de l'Affaire Ménard, paru dans le journal L'Aurore le 4 mars 1898, et simplement intitulé Un bon juge.

(30) Audience du 4 mars 1898 du tribunal correctionnel de Château-Thierry.

(31) Discussion sur le rapport Roux, op. cit., p. 59 ; Robert DOU-

CET, op. cit., p. 92.

(32) Proposition de loi déposée à la chambre des députés par 
Alexandre Millerand le 16 mars 1900.

(33) J.-A. ROUX,

"Discussion..." et

"Rapport...", op. cit., Robert DOUCET, op. cit. ; Camille CHASSIN, La responsabilité pénale et l'extrême misère, discours de rentrée à l'audience solennelle de la cour d'appel de Pau, Pau, GARET, 1900, 28 p.

(34) J.-A. ROUX,

“Discussion...”, op. cit., p. 57.

(35) Id., p. 55.
Cette proposition de loi soumet l'exonération pénale de l'individu qui commet un vol d'objet de première nécessité. La Commission de législation criminelle en charge de préparer la loi demande l'avis consultatif de la Société générale des prisons qui débat sur cette question lors des assemblées générales de la Société du 7 novembre et du 9 décembre 1900. Le vol nécessaire est donc au cœur des discussions, mais au delà de cette simple question, c'est le principe de l'état de nécessité dans son ensemble qui est discuté. Les réflexions tournent autour de trois questions principales : Faut-il ou non introduire la justification du vol par la nécessité ? Faut-il élargir le concept de la nécessité et l'étendre à tous les cas de figure possibles ? Quels critères doivent être présents pour fonder l'état de nécessité ?

Les criminalistes et les théoriciens du droit qui prennent part à ces réflexions ont des avis très partagés sur ces questions. Deux lignes de positions semblent néanmoins se détacher du lot : les partisans de l'introduction de la notion de nécessité dans la loi pénale, eux-mêmes ne s'accordant pas sur la formulation du principe, et les adversaires du rajout de la nécessité dans le Code. Les premiers reconnaissent que le Code pénal présente une insuffisance en ce qui concerne la nécessité, et proposent de rajouter un texte complémentaire, incluant le terme de nécessité, à l'article 64 du Code existant. Seule la formulation du texte en lui-même pose problème. En effet, les uns, emmenés par M. Garçon, sont partisans d'une formule très réduite laissant une certaine liberté d'appréciation aux juges ; les autres, dont J.-A. Roux est le représentant, sont favorables à une définition plus détaillée des cas relevant de l'état de nécessité. Les seconds, principalement des magistrats, considèrent l'ajout de la notion de nécessité comme « inutile » et «dangereux ${ }^{(34)}$ », ils voient dans la théorie du délit nécessaire un "encouragement à la paresse et au vagabondage ${ }^{(35)}$ », un droit au vol.

Le principe de l'état de nécessité en tant que tel semble en majorité bien accepté par les criminalistes ; seule l'application faite de celui-ci au cas du vol apparaît comme un frein, un obstacle à son introduction définitive dans la législation pénale : il fait peur.

La Commission de législation criminelle, après travaux, décide de l'ajout d'un paragraphe supplémentaire à l'article 64 du Code pénal introdui- 
sant l'exonération pénale de l'auteur d'un vol d'objet de première nécessité en cas d'extrême misère : "Il n'y a ni crime, ni délit, ni contravention: $1^{\circ}$ lorsque le prévenu était en état de démence au temps de l'action; $2^{\circ}$ lorsqu'il a été contraint par une force à laquelle il n'a pu résister; $3^{\circ}$ lorsqu'il a accompli l'acte incriminé en état de nécessité, afin de se préserver ou de préserver autrui d'un danger ou d'un dommage présent, imminent, injuste, qu'il n'a pu détourner qu'en exécutant l'acte qui lui est reproché et à condition que le mal causé soit moindre que le mal évité."

Si l'on s'intéresse de plus près à ce texte qui ne sera pas adopté, on s'aperçoit qu'il contient, sous une autre formulation, toutes les conditions requises par le texte actuel du nouveau Code pénal.

Le vol nécessaire et la notion de nécessité provoquent aujourd'hui, comme au XIXème siècle, des réactions vives et passionnées.

L'historien a toute sa place dans une étude qui n'a jusqu'à présent été abordée que du point de vue juridique. Par une approche anthropologique des archives judiciaires, il réintroduit dans cet univers l'élément bumain qu'est le voleur. Ce voleur devient alors un objet d'étude au même titre que la société judiciaire qui reste décidément bien impuissante devant la question du vol nécessaire.

Les quelques exemples mis en avant qui illustrent le vol et sa justification dans la nécessité, ainsi que les discussions des juristes sur ce point, sont autant de pistes de recherches à approfondir pour construire l'histoire d'une notion qui mérite le plus grand intérêt. 